\title{
Rectification of the lateral Casimir force in a vibrating non-contact rack and pinion
}

\author{
Arash Ashourvan and MirFaez Mir|* \\ Institute for Advanced Studies in Basic Sciences, Zanjan, 45195-1159, Iran \\ Ramin Golestanian才 \\ Department of Physics and Astronomy, University of Sheffield, Sheffield S3 7RH, UK
}

(Dated: August 1, 2017)

\begin{abstract}
The nonlinear dynamics of a cylindrical pinion that is kept at a distance from a vibrating rack is studied, and it is shown that the lateral Casimir force between the two corrugated surfaces can be rectified. The effects of friction and external load are taken into account and it is shown that the pinion can do work against loads of up to a critical value, which is set by the amplitude of the lateral Casimir force. We present a phase diagram for the rectified motion that could help its experimental investigations, as the system exhibits a chaotic behavior in a large part of the parameter space.

PACS numbers: 07.10.Cm,42.50.Lc,46.55.+d,85.85.+j
\end{abstract}

As the technological advances lead to miniaturization of mechanical devices, engineers face new challenges that are brought about by the fundamentally different rules that apply at small scales. One of the biggest problems in small machines is the excessive wear of the many surfaces that work in contact with each other, which severely constrains the durability of such machine parts [1]. A particularly attractive idea for overcoming this problem might be to try and exploit the Casimir effect [2, 3] to transduce mechanical forces at small scales between different machine parts that do not have contact with each other. While the classic normal Casimir force between planar boundaries might not be well suited to this task, the lateral Casimir force between corrugated surfaces - which has been predicted [4] and observed [5] recently - might be a better candidate. In an attempt along these lines, we studied the feasibility of a rack and pinion without contact as a mechanical transducer, and found that the coupling provided by the lateral Casimir force can make the pinion stay locked-in with the rack up to surprisingly large velocities even while doing work against an external load [6].

The coupling provided by Casimir force between machine parts is nonlinear, and could lead to complicated dynamics as already demonstrated by Capasso and collaborators using the normal Casimir force [7]. This implies that any experimental realization of such force transduction mechanisms should be carefully guided by theoretical studies of the phase behavior of the system in the space of tunable parameters. For example, the nonlinear coupling could very easily lead to chaos, which is presumably unwanted in such cases and should thus be avoided.

It might also be possible to use the nonlinear properties to our advantage. A particularly interesting case is the possibility of producing a net directed output motion

\footnotetext{
*Electronic address: miri@iasbs.ac.ir

${ }^{\dagger}$ Electronic address: r.golestanian@sheffield.ac.uk
}

(which is non-compact in the parameter space) by using a periodic (compact) input motion. This problem is analogous to swimming [8], and somewhat related to the fluctuation-induced ratchets [9]. While we are primarily interested in directed motion induced by static Casimir force, we note that dynamic Casimir force [4, 10] can also generate motion by controlled emission of photons 11] and getting propulsion from the back-reaction, as exemplified by the vacuum "flying carpet" design proposed in Ref. [12].

Here we study the dynamics of a pinion that is kept at a distance from a vibrating rack, as shown in Fig. 1. The two parts are coupled by the lateral Casimir force between the neighboring surface areas, which is a nonlinear coupling reminiscent of the Josephson coupling in superconductor junctions [4]. We focus on the case of sinusoidal corrugations with a single wavelength, and show that it is possible to get a rectified motion of the pinion. This is a manifestation of a spontaneous symmetry

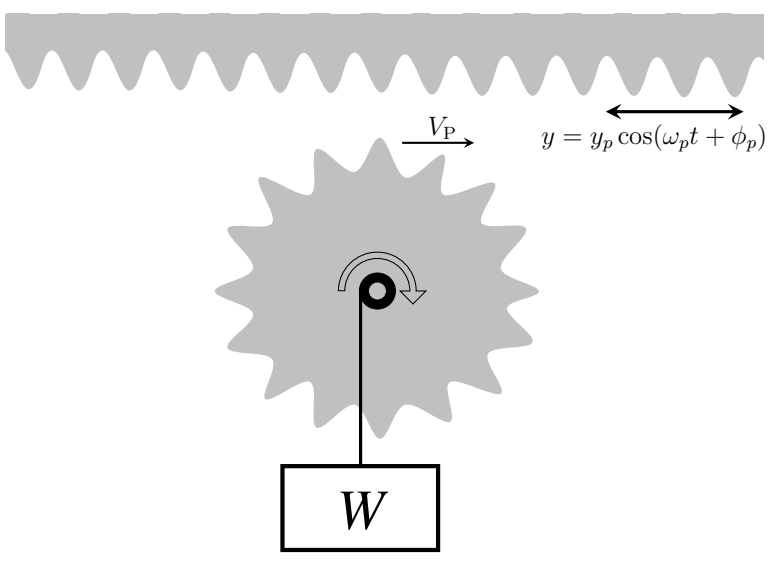

FIG. 1: The schematics of the non-contact rack and pinion, with the rack vibrating laterally. Both the rack and the pinion have sinusoidal corrugations of wavelength $\lambda$. The rectified motion of the pinion will manifest itself in a positive average pinion velocity $V_{\mathrm{P}}$, while working against an external load $W$. 


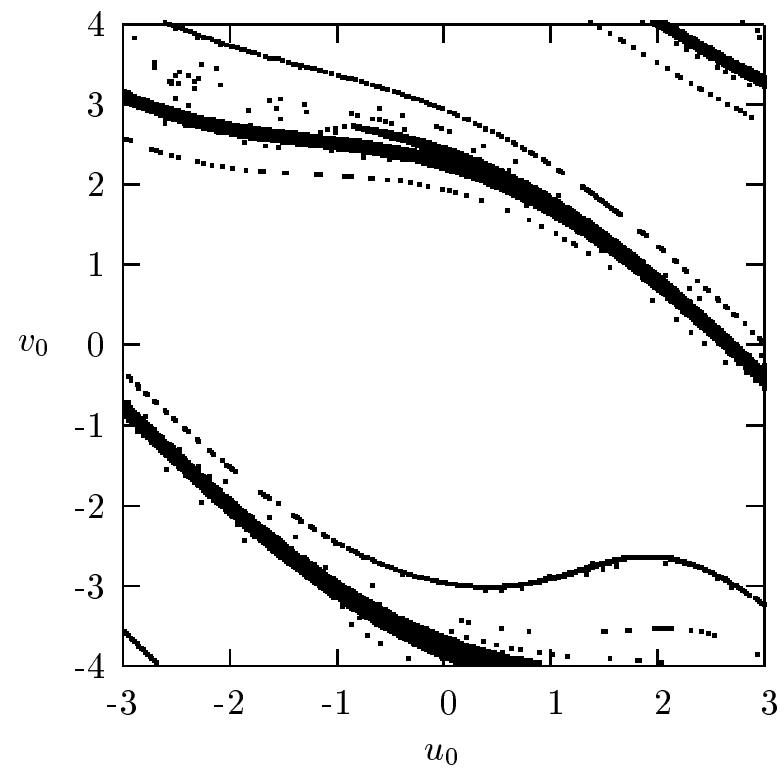

FIG. 2: Basin of attraction for $y_{s}=1.4, \omega_{s}=2 / 3, \phi_{s}=0$, $\epsilon=0.5, m=n=1$, and $w=0.185$. These initial conditions lead to $V_{\mathrm{P}}>0$.

breaking in the system, which is due the inherent nonlinear structure of the dynamics. The average pinion velocity of the rectified motion takes on discrete values and is set by the wavelength of the corrugation $\lambda$ and the frequency of vibration. We have also observed that the system shows a chaotic behavior in a large part of the parameter space. We have studied the effect of external load, and found an upper limit for the load it can work against which is set by the amplitude of the lateral Casimir force. We note that a similar study to ours has been recently performed by Emig, in which he considers vibrations in the normal distance between otherwise stationary corrugated surfaces [13].

If two corrugated surfaces are placed parallel to each other, they experience a lateral Casimir force that tends to displace them so that their "cogs" will face each other to minimize the Casimir energy. The force exerted on the lower plate reads [4]

$$
F_{\text {lateral }}=-F \sin \left[\frac{2 \pi}{\lambda}(x-y)\right]
$$

where $x-y$ is the lateral relative displacement, $\lambda$ is the corrugation wavelength of the two surfaces, and $F$ is the amplitude (see below) [5, 14]. In the setup shown in Fig. 1. we are interested in the dynamics of the pinion of radius $R$ which is subject to a net torque $R F_{\text {lateral }}$ due to the lateral Casimir force, in addition to other forces such as an external load $W$. We can write the equation of motion for the coordinate $x=R \theta$ ( $\theta$ representing the rotation dynamics) as

$$
\frac{I}{R} \frac{d^{2} x}{d t^{2}}=-R F \sin \left[\frac{2 \pi}{\lambda}(x-y)\right]-\frac{\zeta}{R} \frac{d x}{d t}-r W,
$$

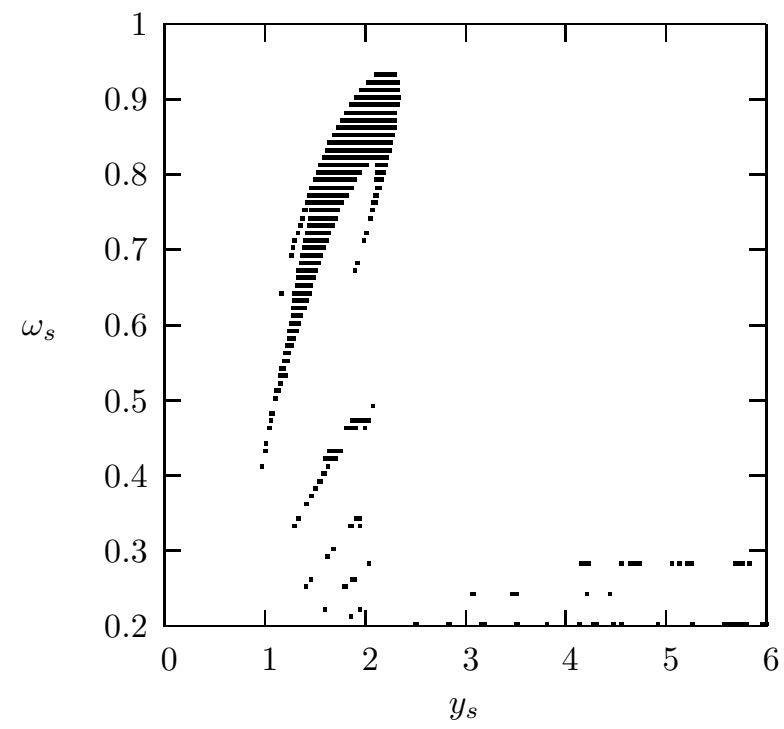

FIG. 3: Values of $y_{s}$ and $\omega_{s}$ which lead to a net rotation of the pinion described by the velocity given in Eq. (9) with $m=n=1$. Here $x_{0}=0, \dot{x}_{0}=0, \epsilon=0.5$, and $w=0.1$.

where $I$ is the moment of inertia about its major axis, and $\zeta$ is the rotational friction coefficient, and $r$ is the torque arm for the external load.

We can study the above nonlinear equation in the phase plane $(u, v=\dot{u})$ where $u \equiv 2 \pi(x-y) / \lambda$, and the rescaled time $\tau$ is measured in units of $T=$ $\sqrt{I \lambda /\left(2 \pi F R^{2}\right)}$, namely $t=T \tau$. We consider harmonic motion of the plate, i.e.

$$
y=y_{p} \cos \left(\omega_{p} t+\phi_{p}\right)
$$

and rewrite Eq. (2) as

$$
\ddot{u}=-\sin u-\epsilon \dot{u}-w+y_{s} \cos \left(\omega_{s} \tau+\phi_{s}\right),
$$

where $\epsilon=T \zeta / I, w=r W /(R F), \omega_{s}=\omega_{p} T$, and

$$
\begin{aligned}
& y_{s}=\left(\frac{y_{p} \omega_{p} I}{F R^{2}}\right)\left[\omega_{p}^{2}+\frac{\zeta^{2}}{I^{2}}\right]^{1 / 2}, \\
& \phi_{s}=\phi_{p}-\tan ^{-1}\left(\frac{\zeta}{I \omega_{p}}\right) .
\end{aligned}
$$

The dynamics of the system is described by Eq. (4) which depends on five (dimensionless) parameters: $\epsilon$ that measures the relative importance of friction to inertia, $w$ that is the external load, and the amplitude $y_{s}$, the frequency $\omega_{s}$, and the initial phase mismatch $\phi_{s}$ of the oscillatory input motion.

Instead of embarking on a full study of the phase diagram of this system, we focus on the specific cases of average rectified motion. Following Refs. [15, 16], we seek for pairs of period steady-state solutions $\left(u^{*}, v^{*}=\dot{u}^{*}\right)$ such that

$$
\begin{aligned}
& u^{*}\left(\tau+2 \pi n / \omega_{s}\right)=u^{*}(\tau) \pm 2 \pi m \\
& v^{*}\left(\tau+2 \pi n / \omega_{s}\right)=v^{*}(\tau)
\end{aligned}
$$


where $m$ and $n$ are integer numbers. We also focus on the upward motion of the load or positive average velocities. The average pinion velocity in this case can be deduced from $\overline{v^{*}}=m \omega_{s} / n$, namely

$$
V_{\mathrm{P}}=\frac{m}{n}\left(\frac{\lambda \omega_{p}}{2 \pi}\right)
$$

The steady-state solutions of Eqs. (17) and (8) are only valid for specific values of the initial conditions $u_{0}$ and $v_{0}$, and therefore the initial values must be tuned to ensure $V_{\mathrm{P}}>0$. In Fig. 2, we have shown the basin of attraction for solutions with $V_{\mathrm{P}}>0$, using $y_{s}=1.4, \omega_{s}=2 / 3$, $\phi_{s}=0, \epsilon=0.5, m=n=1$, and $w=0.185$. The values of the initial conditions $u_{0}$ and $v_{0}$ have been changed by increments of 0.02 across the phase plane.

Equation (9) shows that the pinion velocity for the rectified motion could only acquire distinct values set by the two integers $m$ and $n$, and the overall scale is set by the vibration frequency of the rack $\omega_{p}$ and the wavelength of the corrugations $\lambda$. It is interesting that the driving parameters can be tuned to set the integers $m$ and $n$. Figure 3 shows the parameters $y_{s}$ and $\omega_{s}$ that lead to $m=n=1$, using $x_{0}=0, \dot{x}_{0}=0, \epsilon=0.5$, and $w=0.1$. In these calculations the steps of $y_{s}$ and $\omega_{s}$ are taken to be 0.01 . Throughout the numerical calculations, we noticed that a large part of the parameter space leads to chaotic behavior. These states were unwanted for our purposes, and have been systematically avoided by checking the negativeness of the largest Lyapunov exponent of the system [17].

The external load against which the pinion is doing work puts a significant impediment on the motion and one wonders if there is a critical value for the load that this device can tolerate, similar to the problem of uniformly moving racks [6]. To obtain this limiting value, we can average the equation of motion over the time interval $2 \pi n / \omega_{s}$, which yields $-w-\epsilon \overline{v^{*}}=\overline{\sin u^{*}}$. This result necessitates

$$
W<\frac{R F}{r}\left(1-\epsilon \omega_{s} \frac{m}{n}\right) .
$$

However, this is only an upper bound for the critical load and one needs to do more to find stronger criteria. We can make an attempt towards this end by using the anzats $u^{*} \approx u_{0}+\overline{v^{*}} \tau+\Delta n / \omega_{s} \sin \left(\omega_{s} \tau / n\right)$ and the identity $\exp (i z \cos \beta)=\sum_{k=-\infty}^{\infty} i^{k} J_{k}(z) \exp (i k \beta)$ to expand the sinus in terms of Bessel functions. Using these, a stronger condition of

$$
W<\frac{R F}{r}\left[\left|J_{n}\left(\frac{n \Delta}{\omega_{s}}\right)\right|-\epsilon \omega_{s} \frac{m}{n}\right]
$$

can be obtained. Our numerical calculations with $y_{s}=$ 1.4, $\omega_{s}=2 / 3, \phi_{s}=0, \epsilon=0.5$, and $m=n=1$ show that the upward motion of the load ceases at $w=0.192$, consistent with our analytical estimate of Eq. (11), which yields $w<0.249$ using $J_{1}\left(\Delta / \omega_{s}\right)<0.582$.

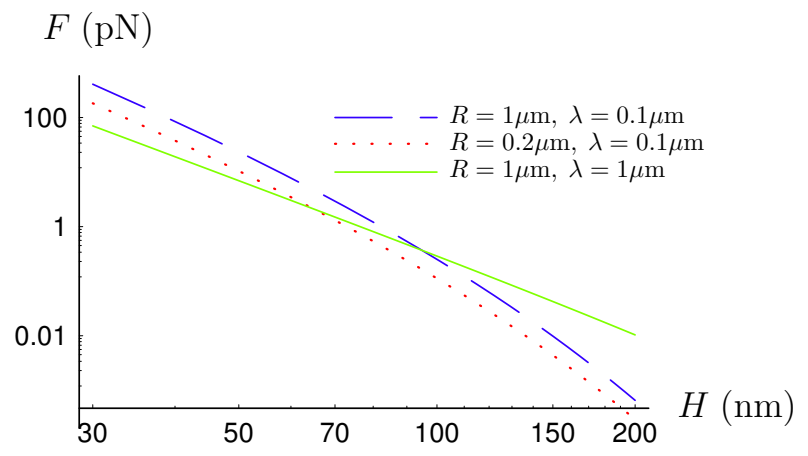

FIG. 4: (Color online) Amplitude of the lateral Casimir force as a function of the gap size for perfect metallic boundaries, corresponding to $a_{1}=a_{2}=10 \mathrm{~nm}$ and $L=10 \mu \mathrm{m}$, for different values of radius and corrugation wavelength. Note that for the case of $R=0.2 \mu \mathrm{m}$ (dotted curve), the larger separations in the plot are approaching the limit of validity of the PFA approximation.

The value of the critical load is essentially set by the amplitude of the lateral Casimir force $F$. We can estimate this quantity for the specific geometry of our rack and cylindrical pinion by using the corresponding Casimir interaction between two parallel corrugated plates and the Proximity Force Approximation (PFA) [18]. For a cylinder of radius $R$ located at a (nearest) distance $H$ from a plate, it has been shown recently that this approximation is reasonably accurate for $H<R$ [19, 20]. Using PFA for the lateral Casimir force between a pinion of length $L$ and corrugation amplitude $a_{1}$ and a rack of corrugation amplitude $a_{2}$, we find

$$
F=\frac{\pi \sqrt{2} \hbar c a_{1} a_{2} L R^{1 / 2}}{\lambda H^{9 / 2}} \int_{1}^{\infty} \frac{d s}{s^{5} \sqrt{s-1}} J\left(\frac{H}{\lambda} s\right)
$$

provided with $a_{1}, a_{2} \ll H$. In this equation $J(u)$ is the coupling function presented in Ref. [14]. This result shows a strong power law behavior at small values of $H$ followed by an exponential decay at large $H$ with the length scale being set by $\lambda$. Figure 4 shows the value of the amplitude $F$ as a function of the shortest distance between the rack and the pinion, for various values of the wavelength and cylinder radius. One can see that the amplitude and thus the maximum load of the system strongly depends on the separation. While typical values for $F$ is in the $\mathrm{pN}$ range for the parameter values we chose, its linear dependence on the length of the pinion $L$ can be used to strengthen the hold of the pinion and make it capable of enduring higher load.

The fact that the system can spontaneously break the symmetry and choose one direction is fascinating and far from trivial. Note that the traditional theory of ratchets always relies on some kind of asymmetry in the structure of the system (such as the two unequal halves of an oscillating saw-tooth potential [9]), to break the symmetry and guide the rectification process. This means that the nonlinear structure of the dynamics of this system, to- 
gether with the presence of the inertial term, has made the spontaneous symmetry breaking possible.

In conclusion, we have studied the dynamics of a setup of rack and pinion that are coupled by the lateral Casimir force, and shown that the coupling can rectify an oscillatory motion in certain areas of the parameter space of the system. This study could shed some light on the novel possibilities that could be available at small scales in systems that are powered by the Casimir force. This method of force transduction could in principle help solve the wear problem in nano-scale mechanics, which makes such studies worthwhile.

This work was supported by EPSRC under Grant EP/E024076/1 (R.G.).
[1] M.P. de Boer, T.M. Mayer, MRS Bull. 26, 302 (2001); R.W. Carpick, Science 313, 184 (2006).

[2] H. B. G. Casimir, Proc. K. Ned. Akad. Wet. 51, 793 (1948).

[3] G. Plunien, B. Müller, and W. Greiner, Phys. Rep. 134, 87 (1986); M. Kardar and R. Golestanian, Rev. Mod. Phys. 71, 1233 (1999); M. Bordag, U. Mohideen, and V. M. Mostepanenko, Phys. Rep. 353, 1 (2001); K. A. Milton, J. Phys. A: Math. Gen. 37, R209 (2004); S. K. Lamoreaux, Rep. Prog. Phys. 68, 201 (2005).

[4] R. Golestanian and M. Kardar, Phys. Rev. Lett. 78, 3421 (1997); Phys. Rev. A 58, 1713 (1998).

[5] F. Chen, U. Mohideen, G.L. Klimchitskaya, and V.M. Mostepanenko, Phys. Rev. Lett. 88, 101801 (2002); Phys. Rev. A 66, 032113 (2002).

[6] A. Ashourvan, M.F. Miri, and R. Golestanian, Phys. Rev. Lett., in press (2007) (preprint quant-ph/0701212).

[7] H.B. Chan, V. A. Aksyuk, R. N. Kleiman, D. J. Bishop and F. Capasso, Phys. Rev. Lett. 87, 211801 (2001).

[8] S. Childress, Mechanics of Swimming and Flying (Cambridge University Press, Cambride, 1981).

[9] A. Ajdari and J. Prost, C. R. Acad. Sci. Paris II 315, 1635 (1993); J. Rousselet, L. Salome, A. Ajdari, and J. Prost, Nature 370, 446 (1994).

[10] G.T. Moore, J. Math. Phys. 11, 2679 (1970); S.A. Fulling, and P.C.W. Davies, Proc. R. Soc. A 348, 393 (1976); M.-T. Jaekel, and S. Reynaud, Phys. Lett. A 167,
227 (1992); G. Calucci, J. Phys. A: Math. Gen. 25, 3873 (1992); P.A. Maia Neto, and S. Reynaud, Phys. Rev. A 47, 1639 (1993); G. Barton and C. Eberlein, Ann. Phys. (N.Y.) 227, 222 (1993); C.K. Law, Phys. Rev. A 49, 433 (1994); V.V. Dodonov, Phys. Lett. A 207, 126 (1995); A. Lambrecht, M.-T. Jaekel, and S. Reynaud, Phys. Rev. Lett. 77, 615 (1996).

[11] F. Miri and R. Golestanian, Phys. Rev. A 59, 2291 (1999); M. Montazeri and M.F. Miri, Phys. Rev. A 71, 063814 (2005).

[12] R. Golestanian, Modern Phys. Lett. B 18, 1225 (2004).

[13] T. Emig, preprint cond-mat/0701641

[14] T. Emig, A. Hanke, R. Golestanian, and M. Kardar, Phys. Rev. Lett. 87, 260402 (2001); Phys. Rev. A 67, 022114 (2003).

[15] V.N. Belykh, N.F. Pedersen, and O.H. Sorensen, Phys. Rev. B 16, 4853 (1977); Phys. Rev. B 16, 4860 (1977).

[16] E.G. Gwinn and R.M. Westervelt, Phys. Rev. A 33, 4143 (1986).

[17] J.H. Argyris, G. Faust, and M. Haase, An Exploration of Chaos (North-Holland, Amsterdam, 1994).

[18] B. Derjaguin, Kolloid-Z. 69, 155 (1934).

[19] T. Emig, R.L. Jaffe, M. Kardar, and A. Scardicchio, Phys. Rev. Lett. 96, 080403 (2006).

[20] M. Bordag, Phys. Rev. D 73, 125018 (2006). 